\title{
Bowel obstruction after laparoscopic and open colon resection for cancer: Results of 5 years of follow-up in a randomized trial
}

\author{
Johnna Schölin • Mark Buunen · Wim Hop · Jaap Bonjer · Bo Anderberg • \\ Miguel Cuesta - Salvadora Delgado - Ainitze Ibarzabal • Marie-Louise Ivarsson • \\ Martin Janson • Antonio Lacy • Johan Lange • Lars Påhlman • Stefan Skullman • \\ Eva Haglind
}

Received: 14 December 2010/Accepted: 28 April 2011/Published online: 11 June 2011

(C) Springer Science+Business Media, LLC 2011

\begin{abstract}
Background Postoperative bowel obstruction caused by intra-abdominal adhesions occurs after all types of abdominal surgery. It has been suggested that the laparoscopic technique should reduce the risk for adhesion formation and thus for postoperative bowel obstruction. This study was designed to compare the incidence of bowel obstruction in a randomized trial where laparoscopic and open resection for colon cancer was compared.

Methods A retrospective analysis was performed, collecting data of episodes of bowel obstruction with or without surgery. Only episodes treated in the hospital where the index surgery took place were included. Data for 786 patients were collected for the 5-year period after cancer surgery.

Results Baseline characteristics for the evaluated laparoscopic $(n=383)$ and open $(n=403)$ groups were
\end{abstract}

J. Schölin · E. Haglind $(\bowtie)$

Department of Surgery, Område 2, Sahlgrenska University

Hospital/Östra, SE-416 85 Göteborg, Sweden

e-mail: eva.haglind@vgregion.se

M. Buunen · J. Lange

Department of Surgery, Erasmus Medical Center,

Rotterdam, The Netherlands

W. Hop

Department of Biostatistics, Erasmus Medical Center,

Rotterdam, The Netherlands

J. Bonjer · M. Cuesta

Department of Surgery, Vrije Universiteit Medical Centre

(VUmc), Amsterdam, The Netherlands

B. Anderberg

Department of Surgery, Capio St: Göran's Hospital,

Stockholm, Sweden comparable. The cumulative obstruction percentages at 5 years for the open and laparoscopic groups were 6.5 and $5.1 \%$ respectively and did not significantly differ from each other. Tumor stage seemed to influence the risk for bowel obstruction: $2.8 \%$ in stage I, $6.6 \%$ in stage II, and $7 \%$ in stage III, but the differences were not significant.

Conclusions This analysis does not support the hypothesis that laparoscopy leads to fewer episodes of bowel obstruction compared with open surgery.

Keywords Colorectal cancer - Surgical technique · Complications · Adhesions - Bowel obstruction . Laparoscopy

Surgical trauma is the cause of more than $90 \%$ of intraabdominal adhesions, and approximately $30 \%$ of patients who undergo major surgery develop postoperative

S. Delgado - A. Ibarzabal · A. Lacy

Department of Surgery, Hospital Clinic, Barcelona, Spain

M.-L. Ivarsson

Department of Surgery, Kungsbacka Hospital, Kungsbacka, Sweden

M. Janson

Department of Surgery, Karolinska University Hospital,

Huddinge, Sweden

L. Påhlman

Department of Surgery, Akademiska Hospital, Uppsala, Sweden

S. Skullman

Department of Surgery, Skövde Hospital, Skövde, Sweden 
adhesion-related complications, such as bowel obstruction, chronic abdominal pain, and infertility $[1,2]$. Up to $80 \%$ of acute intestinal obstructions are caused by adhesions, predominantly involving the small bowel. Intestinal obstruction is associated with high mortality rates [3, 4]. The need for emergency surgical procedures or hospitalization for bowel obstruction is part of the morbidity after all kinds of abdominal surgery, including surgery for colon malignancies.

The Surgical and Clinical Adhesion Research (SCAR) study reported that open colorectal, surgical procedures resulted in the highest risk of adhesion-related readmissions of all types of abdominal surgery [5, 6]. Laparoscopic techniques are associated with less surgical trauma, and one hypothesis concerning laparoscopy as a surgical technique has been that the risk for adhesion formation postoperatively would be lower than after open surgery. Few reports of adhesions after laparoscopy exist [7-9]. Experimental studies have demonstrated that several factors, such as $\mathrm{CO}_{2}$-induced acidosis and metabolic hypoxemia, temperature, and duration of the intervention, are important in the formation of adhesions at laparoscopy [10]. In an experimental model in pigs, it was reported recently that the incidence of intra-abdominal adhesions was lower after laparoscopic compared with open cholecystectomy [11]. It has been reported that open and laparoscopic surgery are associated with comparable risks of adhesion-related readmissions after gynecological surgery [12]. Hypothetically the minimally invasive techniques may result in less adhesion formation compared with open techniques. Adhesions as such are difficult to analyze, but surrogate end points for adhesion-related complications can be admissions for bowel obstruction. The socioeconomic impact of intra-abdominal adhesions is probably underestimated. Surgical techniques resulting in reductions of the frequency of intra-abdominal adhesions and the following complications might be cost-effective, even if more costly initially.

Laparoscopic surgery as a treatment for colorectal cancer was first reported in 1991 by Jacobs et al. [13]. A number of randomized, clinical trials also have published short- and long-term results [14-22]. The primary goal of the colon carcinoma laparoscopic or open resection (COLOR) trial was to compare 3-year cancer-free survival after laparoscopic and open resection for right colon, left colon, or sigmoid cancer, and the sample size was calculated accordingly. Laparoscopic colon resection for colonic cancer results in a smoother immediate postoperative period for the patient and improved health-related quality of life for 2-4 weeks compared with open surgery, at a higher cost for the first 3 months after surgery for hospitals but not in the societal perspective $[14,18,19,23,24]$. In the longer perspective, there was improved [16] or unchanged survival $[17,21,22]$. Based on these trials, laparoscopy as treatment technique for colon cancer should be regarded as a feasible and safe procedure.

This study was designed to compare bowel obstruction due to adhesion formation within the two groups in a randomized trial where laparoscopic and open resection for colon cancer were compared: the COLOR trial. Secondary endpoints were to analyze the risk factors for bowel obstruction and to determine the incidence of reoperation.

\section{Materials and methods}

The COLOR trial is a randomized, international, multicenter trial that compared laparoscopic and open surgery for the treatment of colonic cancer reported elsewhere [15, 19, 21]. Briefly, 1,248 patients were included from 1997 to 2003 and were randomly assigned to laparoscopic or open surgery in a 1:1 fashion. The primary endpoint was cancerfree 3-year survival. Secondary endpoints included cancerfree 5-year survival, 28-day clinical results, morbidity, complications, oncologic outcomes, health-related quality of life, and costs. The patients were followed at least annually during 5 years. Surgeons participating in the inclusion of patients in the COLOR trial had all performed 20 laparoscopic colonic resections before entering the trial. The protocol for the COLOR trial was approved by the appropriate ethics committee of each participating institution before its entry into the trial, and the details of inclusion, exclusion, and consent have been described [12]. Patients were included in the trial according to the inclusion and the exclusion criteria, and of the initially 1,248 included patients 1,083 patients were left for follow-up, as described [19].

\section{Data collection}

Bowel obstruction was not specifically mentioned as an adverse event to note, in the clinical record form (CRF) used for the yearly follow-up visits. Therefore, to study bowel obstruction postoperatively, a specific CRF was designed and sent out to a selection of participating hospitals. This study was undertaken in Swedish (40\% of the trial population) and Dutch hospitals as well as the single largest participating hospital (Hospital Clinic, Barcelona), altogether 15 hospitals, in total 878 of $1,083(81 \%)$ of the study cohort. The follow-up for bowel obstruction was retrospective in nature. Hospital charts were checked, and the study period per patient covered the period of 5 years after index surgery. Each hospital was asked to go through their hospital records for all included patients and to register episodes of hospital stay with the diagnosis bowel obstruction (ICD 10 code K565, K566, or K567) with or 
without operation. The timeframe was from the time of inclusion into the COLOR trial and the following 5 years. This method resulted in documentation of hospitalizations and operations for bowel obstruction at the including hospital. The COLOR database was used for the following data: randomization group, age, gender, body mass index (BMI), ASA, date of surgery, conversion, performed operative procedure, time of last follow-up, and date of death.

Episodes of bowel obstruction due to recurrent disease were not included. The material consisted of reports about bowel obstruction episodes within 5 years of index operation for 878 patients (Table 1). Ninety-two patients, who were reported in this retrospective follow-up but had been excluded from the main COLOR trial in accordance with the protocol or had decided not to participate or were lost to follow-up, were not included in this analysis; 786 patients remained for the analysis.

\section{Statistical analysis}

The cumulative incidence of obstructions during the evaluated period of 5 years after surgery was assessed with use of the Kaplan-Meier method, and the difference between the two treatments arms were compared with the log-rank test. Deaths during follow-up and any losses to follow-up without previous obstructions were considered as censored observations. Multivariate analyses of the incidence of the (first) obstructions were performed using Cox regression. These analyses took into account gender, age, BMI, operative procedure, and stage of disease at operation. All analyses were done in duplicate: one analyzing all obstructions that had occurred and a second one in which obstructions during the first month after the index operation was not counted. Other comparisons between treatments were done with the Mann-Whitney test for continuous or ordered categorical

Table 1 Flow chart of patients included into the COLOR trial by the 15 hospitals that participated in the retrospective follow-up regarding bowel obstruction

\begin{tabular}{lr}
\hline Originally included in main COLOR trial & 965 \\
Reported in follow-up for bowel obstruction & 878 \\
Excluded & \\
Radical index surgery not possible & 50 \\
Benign tumor or no tumor & 20 \\
Other cancer than colon cancer at index surgery & 10 \\
Other violations of inclusion criteria & 8 \\
No follow-up & 4 \\
Total & 92 \\
Remaining for analysis & 786 \\
\hline
\end{tabular}

Patients lost to follow-up during the 5-year period have not been excluded but analyzed until such loss data or the $\chi^{2}$ test, if appropriate. Analyses were conducted by intention-to-treat, and $P=0.05$ (two-sided) was considered the limit of significance.

\section{Results}

Baseline characteristics for the evaluated laparoscopic $(n=383)$ and open group $(n=403)$ were comparable (Table 2). Mean (median) follow-up time of the respective groups was $4.1(5)$ and $4.3(5)$ years $(P=0.11)$. Overall 5 -year survival in the laparoscopic and open group was 72 and $76 \%$, respectively $(P=0.14, \log$-rank test). Seventeen patients in the laparoscopic group and 24 in the open group had at least one obstruction (Table 3). Counting all episodes of bowel obstruction, the cumulative percentages of patients who had at least one episode within 5 years for the open and laparoscopic group were 6.5\% $( \pm 1.3$ standard error) and $5.1 \%( \pm 1.2)$, respectively $(P=0.39$, log-rank test; Fig. 1). The 95\% confidence interval (CI) for the difference (open minus laparoscopic) of 1.4 percentage points at 5 years ranges from -2.1 to $+4.9 \%$.

Multivariate analysis using Cox regression taking into account stage, operative procedure, age, gender, and BMI did not show a significant difference between the randomized groups $(P=0.53)$. Other factors that were analyzed were not significantly related to the occurrence of a

Table 2 Demographics for the patients in the two randomized groups

\begin{tabular}{lccc}
\hline & $\begin{array}{l}\text { Laparoscopic } \\
\text { group }(n=383)\end{array}$ & $\begin{array}{l}\text { Open group } \\
(n=403)\end{array}$ & $P$ value \\
\hline Tumor stage & $89(23 \%)$ & $100(25 \%)$ & 0.86 \\
1 & $161(43 \%)$ & $167(42 \%)$ & \\
2 & $129(34 \%)$ & $131(33 \%)$ & \\
3 & & & \\
Gender & $186(49 \%)$ & $191(47 \%)$ & 0.8 \\
Female & $197(51 \%)$ & $212(53 \%)$ & \\
Male & & & \\
Operative procedure & $196(51 \%)$ & $191(47 \%)$ & 0.58 \\
Right hemicolectomy & $27(7 \%)$ & $31(8 \%)$ & \\
Left hemicolectomy & $147(38 \%)$ & $161(40 \%)$ & \\
Sigmoid resection & $13(3 \%)$ & $20(5 \%)$ & \\
Other & & & \\
BMI (kg/m $\left.{ }^{2}\right)$ & $214(58 \%)$ & $201(52 \%)$ & 0.11 \\
$\leq 25$ & $156(42 \%)$ & $185(48 \%)$ & \\
$>25$ & & & \\
Age (years) & $176(46 \%)$ & $184(46 \%)$ & 0.93 \\
$\leq 70$ & $207(54 \%)$ & $219(54 \%)$ & \\
$>70$ & & & \\
\hline
\end{tabular}

Missing data: tumor stage $(n=9)$, BMI $(n=42)$

Data shown as numbers of patients with percentages in parentheses 
Table 3 Number of patients with none, one, or more episodes of bowel obstruction during 5 years of follow-up, according to randomization group

\begin{tabular}{llllll}
\hline $\begin{array}{l}\text { Number of } \\
\text { episodes }\end{array}$ & All episodes & & \multicolumn{2}{l}{$\begin{array}{l}\text { Episodes requiring } \\
\text { surgery }\end{array}$} \\
\cline { 2 - 3 } & Laparoscopy & Open & & Laparoscopy & Open \\
\hline 0 & $366(369)$ & 379 & & $372(374)$ & 395 \\
& & $(383)$ & & $(396)$ \\
1 & $9(7)$ & $15(13)$ & $8(6)$ & $7(7)$ \\
2 & $5(4)$ & $6(5)$ & $3(3)$ & $0(-)$ \\
3 & $2(2)$ & $3(2)$ & $0(-)$ & $1(0)$ \\
4 & $1(1)$ & $0(-)$ & - & - \\
$\begin{array}{c}\text { Total number of } \\
\text { patients }\end{array}$ & 383 & 403 & 383 & 403 \\
\hline
\end{tabular}

Data are shown as numbers of patients counting all episodes and counting only episodes requiring surgery. Data within brackets denote number of patients not counting episodes within the first month after index surgery

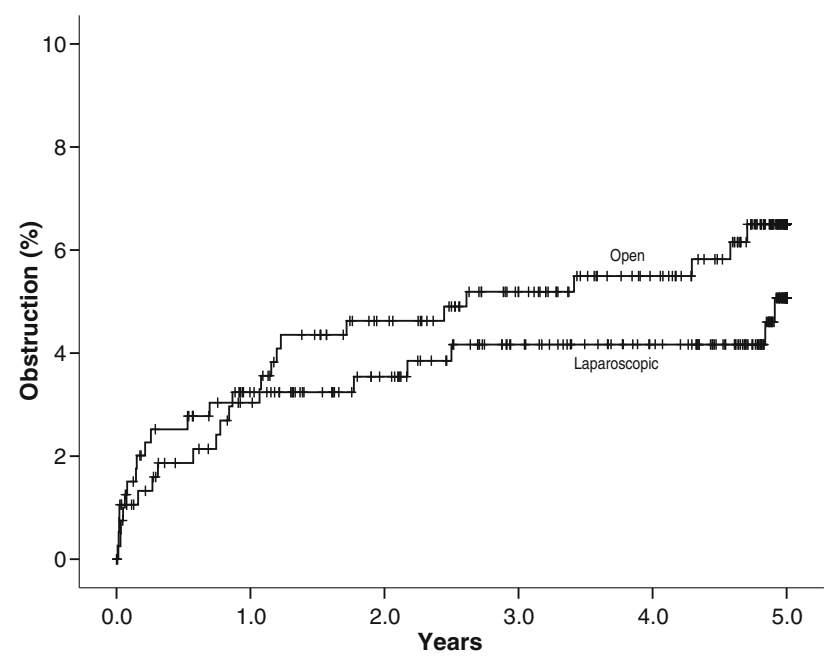

Fig. 1 Kaplan-Meier curves of the cumulative incidence of a first bowel obstruction by randomized treatment $(P=0.39$, log-rank test). Tick marks denote censored observation times

(first) obstruction episode (all $P>0.2$ ). In both randomized groups combined, the cumulative 5-year obstruction rate for stages I, II, and III was 2.8, 6.6 and 7\%, respectively, but this increase was not significant $(P=0.12$; log-rank test for trend).

Disregarding obstructions during the first month after index surgery, similar results were found; the cumulative obstruction percentages at 5 years were $5.6 \%( \pm 1.2)$ in the open group and $4.3 \%( \pm 1.1)$ in the laparoscopic group $(P=0.43$, log-rank test $)$.

The total number of obstruction episodes compared between the two randomized groups did not differ significantly ( $P=0.4$; Mann-Whitney test) or when only episodes occurring after the first postoperative month were analyzed $(P=0.79)$. Bowel obstructions that required surgery did not differ between the groups $(P>0.4)$.

All analyses mentioned above were conducted using intention-to-treat, i.e., patients for whom laparoscopic operation was converted remained in the originally assigned randomized laparoscopic group.

In the laparoscopic group, there were 8 pre- and 72 peroperative conversions. In one case, information about conversion was missing. In the patients who were converted preoperatively, no obstructions occurred. In the peroperatively converted group, five patients suffered obstructive episodes: three patients had one episode and one patient had two episodes. Comparing the episode count between the peroperatively converted and the nonconverted laparoscopic group, no significant difference was found (Mann-Whitney test, $P=0.32 ; P=0.87$ excluding episodes during the first month). The cumulative 5-year incidence rate of a first obstruction for the peroperatively converted and the nonconverted group was 9 and 4.3\%, respectively $(P=0.19$, log-rank test $)$.

\section{Discussion}

The reason for performing this retrospective follow-up regarding bowel obstruction was that this complication was not included when the CRFs for annual follow-up were designed. Because data on this specific complication can easily be found in the hospital files with high validity, the steering committee for the trial decided that the opportunity to analyze bowel obstruction should not be missed, albeit as a retrospective study. The population was defined, the external validity for a large part of the population was known [25], and the trial was randomized. Altogether these factors made an analysis more important, because there is a lack of high-quality data on bowel obstruction after laparoscopic surgery.

Because this follow-up was retrospective, the fact that not all hospitals participated should not influence results. The majority $(75 \%)$ of included patients were involved in this survey. For each participating hospital in this study, all included patients were actively looked for in the records for that hospital. The episodes of bowel obstruction missed in the population were those where the patient had been treated in another hospital then the hospital where the index cancer surgery had been performed. There is no reason to believe that this should differ between the two randomized groups: laparoscopic and open surgery.

There was no difference in bowel obstruction comparing laparoscopic to open surgery, irrespective of whether bowel obstruction episodes later than 1 month after index surgery or all episodes after discharge were included. Likewise, including only procedures that were performed 
laparoscopically without conversion did not change this result. It does not seem likely that a larger sample size would change the result resolutely. One possible explanation, at least in part, could be the relative lack of experience for many of the participating surgeons, particularly early in the inclusion phase, which not only would result in conversions but also could result in a technique that did not fulfil the "minimally invasive" concept [26]. However, it is possible to argue that participating surgeons by learning laparoscopic technique where dissection in the correct anatomical planes is essential for success, used this technique also in the open cases, and thus the patients in the openly operated group also benefited from an atraumatic surgical technique. The result of this study is similar to the recent report from the CLASICC trial-another of the randomized trials of laparoscopic versus open surgery, but for colorectal cancer, with similar protocol and a somewhat smaller sample [27].

In conclusion, the results of this study of a randomized population did not confirm the hypothesis that laparoscopy leads to fewer episodes of bowel obstruction or fewer operations for bowel obstruction compared with open surgery, during a 5-year follow-up after resection for colon cancer.

Acknowledgments The authors express their sincere gratitude to RN Elisabet Lindholm, Dr. Gunnar Edlund, Dr. Johan Dalen, Dr. Owe Lundberg, and Dr. Ulf Kressner who all made significant contributions to make this report possible. This work was supported by grants from Sahlgrenska University Hospital, Sahlgrenska Academy at University of Gothenburg and the Swedish Cancer Foundation.

Disclosures Dr. Lacy is a Consultant for Covidien and for Olympus Medical. Dr. Buunen presently works as Senior Medical Advisor for Grunenthal $\mathrm{GmbH}$, Aachen. Authors Anderberg, Bonjer, Cuesta, Delgado, Haglind, Hop, Ibarzabal, Ivarsson, Janson, Lange, Påhlman, Skullman, and Schölin have no conflicts of interest or financial ties to disclose.

\section{References}

1. Menzies D, Ellis H (1990) Intestinal obstruction from adhesions-How big is the problem? Ann R Coll Surg Engl 72:60-63

2. Ellis H, Moran BJ, Thompson JN, Parker MC, Wilson MS, Menzies D, McGuire A, Lower AM, Hawthorn RJ, O'Brien F, Buchan S, Crowe AM (1999) Adhesion-related hospital readmissions after abdominal and pelvic surgery: a retrospective cohort study. Lancet 353:1476-1480

3. Chou NH, Mok KT, Wang BW, Chang HT, Hsu PI (2003) Risk factors of mortality after surgical treatment of intestinal obstruction in patients having prior laparotomy for non-malignancy. J Chin Med Assoc 66:741-746

4. Parker MC, Wilson MS, van Goor H, Moran BJ, Jeekel J, Duron JJ, Menzies D, Wexner SD, Ellis H (2007) Adhesions and colorectal surgery-call for action. Colorectal Dis 9(Suppl 2):66-72

5. Parker MC, Wilson MS, Menzies D, Sunderland G, Thompson JN, Clark DN, Knight AD, Crowe AM (2004) Colorectal surgery: the risk and burden of adhesion-related complications. Colorectal Dis 6:506-511

6. Parker MC, Wilson MS, Menzies D, Sunderland G, Clark DN, Knight AD, Crowe AM (2005) The SCAR-3 study: 5-year adhesion-related readmission risk following lower abdominal surgical procedures. Colorectal Dis 7:551-558

7. Milad MP, Escobar JC, Sanders W (2007) Partial small bowel obstruction and ileus following gynecologic laparoscopy. J Minim Invasive Gynecol 14:64-67

8. Tsao KJ, St Peter SD, Valusek PA, Keckler SJ, Sharp S, Holcomb GW III, Snyder CL, Ostlie DJ (2007) Adhesive small bowel obstruction after appendectomy in children: comparison between the laparoscopic and open approach. J Pediatr Surg 42:939-942 (discussion 942)

9. Rosin D, Zmora O, Hoffman A, Khaikin M, Bar Zakai B, Munz Y, Shabtai M, Ayalon A (2007) Low incidence of adhesionrelated bowel obstruction after laparoscopic colorectal surgery. J Laparoendosc Adv Surg Tech A 17:604-607

10. Bergstrom M, Falk P, Park PO, Holmdahl L (2008) Peritoneal and systemic $\mathrm{pH}$ during pneumoperitoneum with $\mathrm{CO}_{2}$ and helium in a pig model. Surg Endosc 22:359-364

11. Cainzos M, Rodriguez-Segade F, Martinez-Castro J, Prieto D, Becker MR, Aneiros F, Cortes J (2006) Intra-abdominal adhesions after open and laparoscopic cholecystectomy: an experimental model. J Laparoendosc Adv Surg Tech A 16:108-112

12. Lower AM, Hawthorn RJ, Clark D, Boyd JH, Finlayson AR, Knight AD, Crowe AM (2004) Adhesion-related readmissions following gynaecological laparoscopy or laparotomy in Scotland: an epidemiological study of 24046 patients. Hum Reprod 19:1877-1885

13. Jacobs M, Verdeja JC, Goldstein HS (1991) Minimally invasive colon resection (laparoscopic colectomy). Surg Laparosc Endosc 1:144-150

14. Delgado S, Lacy AM, Filella X, Castells A, Garcia-Valdecasas JC, Pique JM, Momblan D, Visa J (2001) Acute phase response in laparoscopic and open colectomy in colon cancer: randomized study. Dis Colon Rectum 44:638-646

15. Hazebroek EJ (2002) COLOR: a randomized clinical trial comparing laparoscopic and open resection for colon cancer. Surg Endosc 16:949-953

16. Lacy AM, Garcia-Valdecasas JC, Delgado S, Castells A, Taura P, Pique JM, Visa J (2002) Laparoscopy-assisted colectomy versus open colectomy for treatment of non-metastatic colon cancer: a randomised trial. Lancet 359:2224-2229

17. Clinical Outcomes of Surgical Therapy Study Group (2004) A comparison of laparoscopically assisted and open colectomy for colon cancer. N Engl J Med 350:2050-2059

18. Guillou PJ, Quirke P, Thorpe H, Walker J, Jayne DG, Smith AM, Heath RM, Brown JM (2005) Short-term endpoints of conventional versus laparoscopic-assisted surgery in patients with colorectal cancer (MRC CLASICC trial): multicentre, randomised controlled trial. Lancet 365:1718-1726

19. Veldkamp R, Kuhry E, Hop WC, Jeekel J, Kazemier G, Bonjer HJ, Haglind E, Pahlman L, Cuesta MA, Msika S, Morino M, Lacy AM (2005) Laparoscopic surgery versus open surgery for colon cancer: short-term outcomes of a randomised trial. Lancet Oncol 6:477-484

20. Jayne DG, Guillou PJ, Thorpe H, Quirke P, Copeland J, Smith AM, Heath RM, Brown JM (2007) Randomized trial of laparoscopic-assisted resection of colorectal carcinoma: 3-year results of the UK MRC CLASICC Trial Group. J Clin Oncol 25:3061-3068

21. Buunen M, Veldkamp R, Hop WC, Kuhry E, Jeekel J, Haglind E, Pahlman L, Cuesta MA, Msika S, Morino M, Lacy A, Bonjer HJ (2009) Survival after laparoscopic surgery versus open surgery for colon cancer: long-term outcome of a randomised clinical trial. Lancet Oncol 10:44-52 
22. Bonjer HJ, Hop WC, Nelson H, Sargent DJ, Lacy AM, Castells A, Guillou PJ, Thorpe H, Brown J, Delgado S, Kuhrij E, Haglind E, Pahlman L (2007) Laparoscopically assisted vs open colectomy for colon cancer: a meta-analysis. Arch Surg 142:298-303

23. Janson M, Bjorholt I, Carlsson P, Haglind E, Henriksson M, Lindholm E, Anderberg B (2004) Randomized clinical trial of the costs of open and laparoscopic surgery for colonic cancer. $\mathrm{Br} \mathbf{J}$ Surg 91:409-417

24. Janson M, Lindholm E, Anderberg B, Haglind E (2007) Randomized trial of health-related quality of life after open and laparoscopic surgery for colon cancer. Surg Endosc 21:747-753

25. Janson M, Edlund G, Kressner U, Lindholm E, Pahlman L, Skullman S, Anderberg B, Haglind E (2009) Analysis of patient selection and external validity in the Swedish contribution to the COLOR trial. Surg Endosc 23:1764-1769

26. Kuhry E, Bonjer HJ, Haglind E, Hop WC, Veldkamp R, Cuesta MA, Jeekel J, Pahlman L, Morino M, Lacy A, Delgado S (2005) Impact of hospital case volume on short-term outcome after laparoscopic operation for colonic cancer. Surg Endosc 19: 687-692

27. Taylor GW, Jayne DG, Brown SR, Thorpe H, Brown JM, Dewberry SC, Parker MC, Guillou PJ (2010) Adhesions and incisional hernias following laparoscopic versus open surgery for colorectal cancer in the CLASICC trial. Br J Surg 97:70-78 